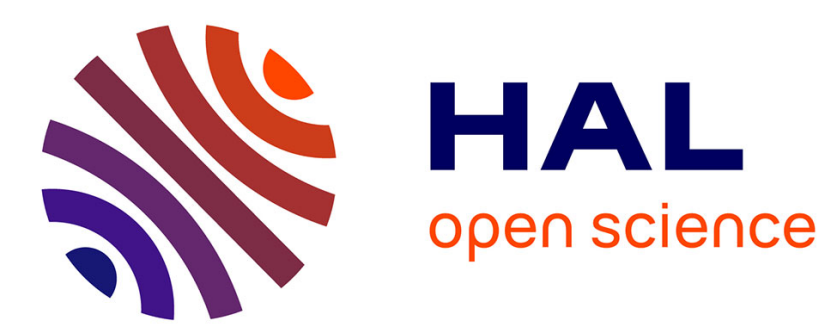

\title{
Highlights from Faraday Discussion on Nanoparticles with Morphological and Functional Anisotropy, Glasgow, UK, July 2015
}

\author{
Benjamin Abecassis, D. Martin A. Buzza, Qijun Song
}

\section{To cite this version:}

Benjamin Abecassis, D. Martin A. Buzza, Qijun Song. Highlights from Faraday Discussion on Nanoparticles with Morphological and Functional Anisotropy, Glasgow, UK, July 2015. Chemical Communications, 2016, 52 (89), pp.13060-13067. 10.1039/c6cc90471h . hal-02995339

\section{HAL Id: hal-02995339 \\ https://hal.science/hal-02995339}

Submitted on 9 Nov 2020

HAL is a multi-disciplinary open access archive for the deposit and dissemination of scientific research documents, whether they are published or not. The documents may come from teaching and research institutions in France or abroad, or from public or private research centers.
L'archive ouverte pluridisciplinaire HAL, est destinée au dépôt et à la diffusion de documents scientifiques de niveau recherche, publiés ou non, émanant des établissements d'enseignement et de recherche français ou étrangers, des laboratoires publics ou privés. 
Highlights from Faraday Discussion on nanoparticles with Morphological and Functional Anisotropy, Glasgow, UK, July 2015.

\author{
Benjamin Abécassis ${ }^{1,2}$, D. Martin A. Buzza ${ }^{3}$, Qijun Song ${ }^{4}$ \\ 1: Laboratoire de Physique des Solides, CNRS, Université Paris-Sud, Univ. Paris Saclay, Bâtiment \\ 510, 91405, Orsay, France.
}

2: Laboratoire de Chimie, CNRS, École Normale Supérieure de Lyon, 46, Allée d'Italie, F-69364 Lyon

3: Department of Physics \& Mathematics, University of Hull, Hull, United Kingdom

\title{
4: School of Chemical and Material Engineering, Jiangnan University
}

1800 Lihu Road, Wuxi, 214122, China

\section{Introduction}

On july the $4^{\text {th }} 2016$, around 120 delegates from all around the world reunited at the technology and innovation centre of the University of Strathclyde in Glasgow to attend the Faraday Discussions meeting on Nanoparticles with Morphological and Functional Anisotropy. Such conferences have been taking place in the UK for more than 100 years and are known for their distinctive format. Instead of long talks followed by short discussions with the speaker, Faraday discussions dedicate most of the time to the interaction between the speaker and the audience. Before the conference, papers presenting new results were selected by the scientific committee and peer-reviewed. The delegates receive the pre-prints several weeks before the conference and hence have time to read the papers in detail so that they have a precise idea of what is going to be presented during the conference. The conference was divided in 4 different thematically focused sessions. For each session, time was separated in two slots where 3 to 4 speakers presented their work in 5 minutes. Afterwards, more than 2 hours were dedicated to questions and answers which where all recorded and will be published later. In this report, we give a brief overview of the talks and social events during the conference. The discussions are covered in a different paper.

The conference started at noon and, after having finished their lunch, the attendees listened to Prof Liz-Marzán, the chair of the conference, who explained the principle of the conference and detailed the composition of the scientific and organization committee. He also introduced the invited introductory speaker, Pr Catherine J. Murphy who kicked off the conference.

\section{Introductory Lecture}

Pr Catherine J. Murphy (University of Illinois at Urbana Champaign, USA) gave the introductory lecture entitled "seed mediated growth of gold nanorods: towards nano rod matryoshkas". In this stimulating talk, she dealt with different topics of great interest for the conference attendees. She started to recall that Michael Faraday, who gave his name to these conferences, was actually the first person to have synthesized gold colloids and that these red dispersions can still be seen at the Royal Institution in London. Since then, important progresses have been made and control over nanoparticle anisotropy is nowadays obtained routinely. Prof Murphy defined in a broad manner anisotropy and stressed that several different mechanisms could lead to anisotropic nanomaterials such as the presence of a template, coalescence and oriented attachment of primary nanocrystals, defect or dislocation driven growth or preferential adsorption and minimization of surface energy. She also 
emphasized that these mechanisms could operate simultaneously as recently shown using in situ environmental transmission electron microscopy.

Prof Murphy then discussed two important topics in the emergence of anisotropy in seed mediated metal nanoparticle synthesis. The use of structure directing agent is now widely recognized as a crucial factor in order to obtain anisotropic nanoparticles. In the case of gold nanorods, the bromide counter-ion of the CTAB surfactant is of particular importance. Another important factor is the salt reduction kinetics which has to be slow enough to allow anisotropy to develop. In order to probe this aspect in more details, Prof Murphy's group performed electrochemistry experiments which showed that, surprisingly, CTAB is redox active. In the studied system, hydroquinone was used as the main reducing agent and the redox activity of hydroquinone and CTAB were found to be of the same order of magnitude, stressing $\mathrm{CTAB}$ may not play a single structure directing agent role in the gold nanorod synthesis.

The lecture was concluded by evoking recent efforts in Prof Murphy's lab to synthesize complex layered structures composed of gold and silica. The goal here is to tune the position of the plasmon peak by growing successive silica and gold shells on an initial gold nanorod. The absorbance spectra of these Matrioshkas structures have been calculated theoretically using electromagnetic simulations and, as stated frankly by Prof Murphy, everything works fine as far as theory is concerned. Of course, nanoparticles with as many as 17 successive shells still represent a huge challenge for nanoparticle chemists! If it is feasible to synthesize core/shell gold/silica nanorods (though the parameter window where reasonably pure products are obtained is narrow), adding another gold layer on the silica surface is still challenging. In the conclusion of her talk, Prof Murphy stated current challenges in the synthesis of anisotropic metallic nanoparticles including the scaling-up of current methods, the difficulty of achieving large aspect ratios and the fact that mechanistic studies should be deepened to disentangle the different phenomenon leading to a symmetry breaking.

\section{Session 1 : Janus and Patchy Nanoparticles}

The first session of the conference, chaired by Prof Axel H.E. Muller, was dedicated to Janus and patchy nanoparticles. Both synthetic aspects (Reguera, Gracias, Duguet) and self-assembly (Granick, Synytska) of Janus particles were discussed in this session.

The first talk was given by Prof Steve Granick (IBS Center for Soft and living Matter, South Korea), who presented recent work on self-organization of Janus particles under the effect of an electric field (DOI: $10.1039 / \mathrm{c} 6 \mathrm{fd} 00077 \mathrm{k})$. The two facets of the Janus particles are covered with materials of different polarizabilities inducing an imbalance which confers a self-propelling character to the particles through induced charge electrophoresis. The interaction potential between the particles is also tuned by the electric field through the magnitude of the dipolar interaction. This delicate control of both the particle motility and the inter-particle interaction through the electric field yields a very rich phase diagram with particles organizing into chains, clusters or moving collectively in the same direction like swarms. Original structures like chiral spirals which rotates clock-wise or counter clockwise were also observed in some cases.

Dr Javier Reguera (CIC biomaGUNE, San Sebastian, Spain) presented recent results on the synthesis of Janus plasmonic-magnetic nanostars (DOI: 10.1039/c6fd00012f ). To obtain such bi-functional nanoparticles, gold-iron oxide heterodimers are first synthesized in solution. A gold nanostar is then grown on the gold moiety which serves as a seed. The growth of the nanostar induces a shift towards longer wavelengths of the plasmon band. Advanced electron microscopy techniques such as HAADF and tomography were used to characterize the particles. Finally, the relevance of particles in applications such as SERS was proven. Interestingly, the authors took advantage of the magnetic 
character of the particles to separate them using a magnet before the SERS measurement showing a nice synergy between the two properties.

Blending bottom-up to top-bottom approaches, Prof David Gracias (Johns Hopkins university, Baltimore, USA) talked about self-folding nanostructures (DOI: 10.1039/c6fd00021e). Using both Electron Beam Lithography (EBL) or Nanoimprint Lithograph (NIL), Prof Gracias and his group not only create $2 \mathrm{D}$ structures with a resolution close to the atomic scale but also use a variety of forces such as capillarity or swelling and shrinking to induce the folding of these nanostructures into 3D objects. For example, $500 \mathrm{~nm}$ self-folded cube shaped boxes made of $\mathrm{Ni} / \mathrm{Sn}$ and patterned with $\mathrm{Au}$ and $\mathrm{Al}_{2} \mathrm{O}_{3}$ could be formed due to the bi-axial strain during $\mathrm{Sn}$ reflow. The advantages and drawbacks of the different techniques were then discussed.

After the discussion on these papers and and afternoon tea break, the talks resumed and since Dr Velikov could not present his paper, the second part of the session started with the presentation by Dr Alla Synytska (Leibniz Institute of Polymer Research, Dresden, Germany) on the self-assembly of colloidal particles (DOI: 10.1039/c6fd00008h). Silica nanoparticles (100-1000 nm) were synthesized and coated with two types of poly-electrolytes yielding particles with either positive or negative overall charges. Afterwards, the two types of oppositely charged particles were mixed and the selfassembled structures were observed as a function of the concentration ratio between the positive and negative particles. The size difference between the two types of particles was also an important parameter. Various types of structures such as raspberry micro clusters (more likely when small particles are in excess) or dumbbell configurations were observed and rationalized taking into account inter-particle forces, paving the way to even more complex structures.

In the last talk of the session, Prof Etienne Duguet (University of Bordeaux, France) focused on the synthesis of complex gold/silica nanostructures (DOI: 10.1039/c6fd00022c). The ultimate goal of the research presented is to produce colloids with a tetrahedral symmetry which could then self-assemble into a diamond lattice which possess special optical properties, e.g., a 3D photonic bandgap. To achieve this, silica particles with four dimples are first synthesized by making a polystyrene/silica tetrapod followed by dissolution of the polymer. Once this is achieved, gold moieties have to be grown in each of the four "cradles". To do so, Prof Duguet and his team took advantage of polystyrene bumps which remain at the bottom of each dimple and functionalized the polymer with a thiol group in order to attach small gold nanoparticles through a strong and specific sulphur-gold bond. Going a step further, attempts were made to use these small particles as seeds in order to yield larger gold nanoparticles. Unfortunately, though different strategies were tried, only moderate success was achieved. Mild reduction conditions and successive addition of gold precursors yielded the best results, opening interesting perspectives of these highly symmetrical objects in metamaterial and plasmonic applications.

\section{Flash Poster presentations, poster session and wine reception}

After the discussion, flash presentations gave the opportunity to some of the poster presenters to highlight their results using one slide within 30 seconds. Of course, the speakers had a hard time explaining their work in depth but these flash presentations drew the interest of numerous attendees who could have a longer explanation during the poster session which took place immediately afterwards. Wine and beer were served during the poster session and groups of scientist gathered in front of the posters to discuss the science presented mostly by junior researchers.

\section{Session 2 Anisotropic Nanoparticles}


The second session took place on the morning of 5 July with Prof.Alberto Striolo (University College London, UK) as session chair. The session's main topic revolved around anisotropic nanoparticles, including novel methods for their synthesis (Lopez-Quintela, Roig), characterisation (Peddis) and assembly into functional materials on both the mesoscale (Kumacheva) and macroscale (Kotov, Konig).

The session was kicked off by Prof. Nicholas Kotov (University of Michigan, USA) who, due to unforeseen circumstances, delivered the paper by Skype. The paper discussed how the optical anisotropy of individual chiral nanoparticles (NP) could be amplified into a macroscopic system by assembling NP thin films using the layer-by-layer assembly method (LBL) (DOI:

10.1039/c6fd00064a). Specifically, chiral thin films were prepared using negatively charged CdS nanoparticles (synthesised using L-or D-cysteine respectively as chiral stabilising agents) and positively charged polyelectrolytes as building blocks. Interestingly these authors found that the circular dichroism (CD) bands associated with the chirality of the NP cores were conserved when the NPs were assembled into the thin films, while the CD bands associated with the chiral stabilising agents was reversed. The latter effect was attributed to the interaction of these surface ligands with the polyelectrolyte matrix.

Dr Tobias Konig (Leibniz-Institut fur Polymerforschung Dresden, Germany) continued along the theme of translating nanoparticle properties to macroscopic behaviour, but shifting the focus this time to creating magnetic metasurfaces through an ingenious template-assisted assembly of gold nanorods onto a gold film (DOI: 10.1039/c6fd00013d). Specifically, these authors first created a linear array of gold nanorods by dip coating the nanorod solution onto a wrinkled template with a well-defined periodicity. The linear array was then deposited onto a gold film using a wet-contact printing method. Gold nanorods with flat faces were used to maximize the plasmonic coupling between the nanorods and the gold films and exquisite control of the particle-to-film distance was achieved by controlling the thickness of the capping agent on the gold nanorods. The hybridisation of the transverse electric mode of the nanorod with the substrate creates a transverse magnetic mode in the visible spectrum whose resonant frequency can be tuned by changing the particle-to-film distance. In subsequent discussions, it was further clarified that it was particularly important to control the lateral distance between rods in order to prevent the hybridisation of transverse magnetic modes between rods (hence the use of wrinkled substrates with well-defined periodicity) but the control of the longitudinal distance between rods was less critical.

Dr Davide Peddis (ISM-CNR, Italy) due to unforeseen circumstances, also delivered his paper by Skype. The paper discussed a novel tomographic method based on transmission electron microscopy (TEM) to study the 3D morphology of magnetic nanoparticles synthesised by magnetostatic bacteria (MTB) known as Magnetospirillum gryphiswaldense (DOI: 10.1039/c6fd00059b). These bacteria apparently use their internal magnetic nanoparticles as a navigation tool by following the Earth's magnetic field. The main take home message from the paper is that $3 \mathrm{D}$ shape has a big effect on the properties of magnetic nanoparticles and the statistical distribution of shape therefore needs to be taken into account when modelling the collective behaviour of an ensemble of particles.

After the morning coffee break, the session resumed with a beautiful paper by Prof Eugenia Kumacheva (University of Toronto, Canada) on the assembly of patchy and non-patchy metal nanoparticles into linear, polymer-like assemblies ("nanopolymers") (DOI: 10.1039/c6fd00057f). The first part of the paper focussed on the assembly of gold nanorods end functionalised with polymer where linear assembly was triggered by changing the solvent quality for the polymer to induce hydrophobic interactions between the polymer patches. By exploiting the powerful analogy between this process and conventional step-growth polymerisation reactions, the authors were able to gain fine control over the chain-length ("degree of polymerisation") of the nanopolymers. In the second part of the paper, the authors showed that surprisingly, under the right conditions, nanoparticles (specifically 
spheres and cubes) uniformly coated with polymer could also assemble into linear assemblies. This unexpected result could be explained by a balance between attractive hydrophobic forces between nearest neighbour and repulsive electrostatic forces between next nearest neighbour nanomers. In the lively discussion that ensued, a number of variations around the nanopolymer theme were discussed and suggested (many of which are already being pursued by the authors), including the creation of branched nanopolymers, block co-nanopolymers and stimuli responsive nanopolymers (by using responsive polymers for the polymer patches).

In the final two papers of the session, novel methods for synthesising anisotropic NPs were discussed. Prof Arturo Lopez-Quintela (University of Santiago de Compostela, Spain) discussed a new seed mediated method for synthesising gold nanorods by using gold clusters (rather than silver ions) as a catalyst for the reaction (DOI: 10.1039/c6fd00015k). The gold clusters were created with the same recipe as for the gold seeds, but using shorter reaction times. The use of the gold clusters confirms the important role played by clusters in the growth of gold nanorods which was previously postulated but not proven.

Dr Anna Roig (ICMAB-CSIC, Spain) followed with a paper about a novel microwave assisted method for synthesising gold nanotriangles and hexagons decorated with superparamagnetic iron oxide nanoparticles (SPIONS) (DOI: 10.1039/c6fd00028b). The SPIONS could easily be removed afterwards using a dilute acidic solution. Such composite plasmonic/magnetic nanoparticles could have novel applications in bio-sensing, medical imaging or therapeutics.

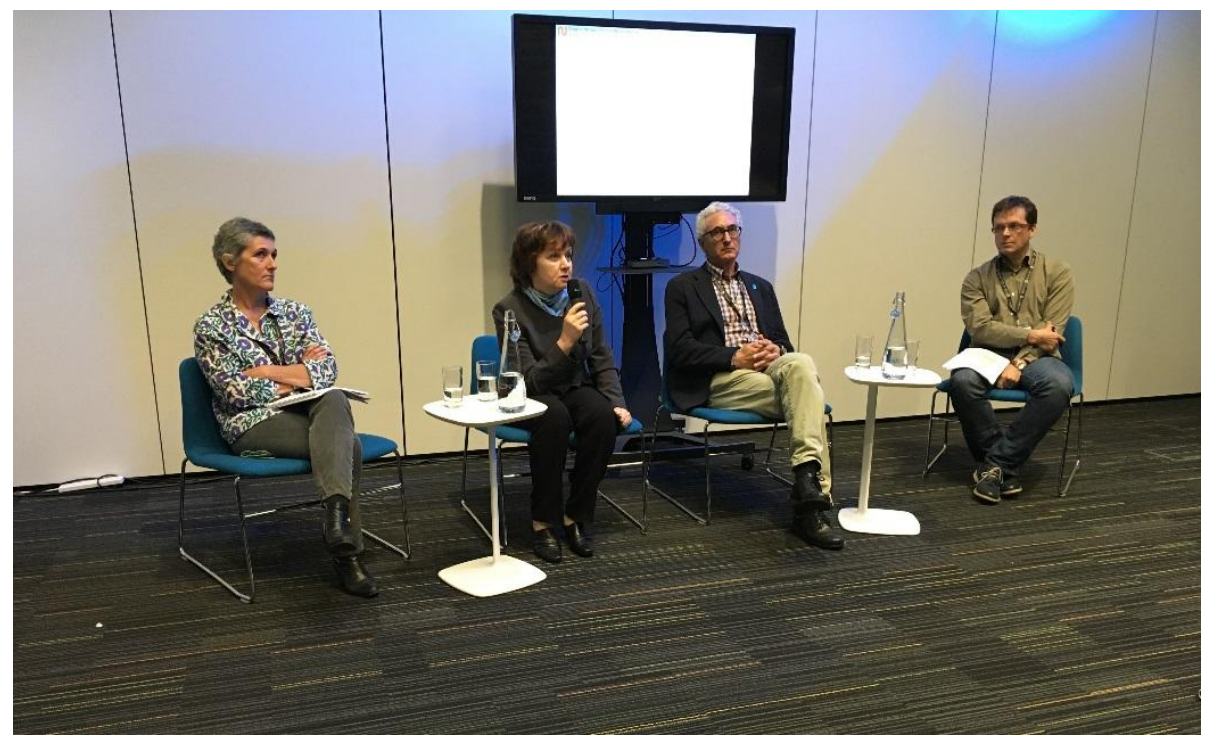

Fig.1: Some of the participants of the second session. From left to right, Dr Anna Roig, Prof Eugenia Kumacheva, Prof Arturo Lopez-Quintela, Prof Alberto Striolo. Photo courtesy of Prof Luis LizMarzán

\section{Session 3 Particles at Liquid Interfaces}

The third session took place in the afternoon of 5 July, with Prof. Joerg Lahann (University of Michigan, USA) as session chair. The session encompassed a broad range of topics, including particle stabilised emulsions (Binks, Sicard), the wetting behaviour of particles at liquid interfaces (Stocco), the synthesis of novel surface active and end functionalised particles (Vermant, Gonzalez Solveyra, Thill) and a novel spray-assisted assembly method for nanorods (Pauly).

The session was kicked off by Prof Bernie Binks (University of Hull, UK) who described a novel method for stabilising emulsions based on using polyelectrolyte complexes (PECs) formed by oppositely charged water-soluble polymers of similar molecular weights (DOI: 10.1039/c6fd00011h). 
The formation of the PECs was crucial for emulsion stability as either polymer alone was incapable of stabilising the emulsion. The PECs were first studied in aqueous solutions and their size suggest that they are formed by a pair of oppositely charged polymers. Emulsion stabilisation by the PECs was achieved by close-packed particle layers at the drop interface and particle aggregation in the continuous phase.

Dr Francois Sicard (University College London, UK) continued the theme of particle stabilised emulsions by studying the stability of these systems within the framework of dissipative particle dynamics (DPD), a mesoscale computer simulation method (DOI: 10.1039/c6fd00055j). Specifically, the authors considered the early stage coalescence of water drops in decane stabilised by homogeneous and Janus nanoparticles with different shapes (spherical, ellipsoidal) and wettability. Different droplet collision speeds were also studied using Adiabatic Biased Molecular Dynamics simulations (slow collision) and pulling simulations (fast collision). Coalescence was found to be initiated when a nanoparticle from one water droplet first touches the other water droplet, and the specific stabilisation mechanism was found to strongly depend on the characteristics of the stabilising nanoparticles and droplet collision speed.

The paper by Dr Antonio Stocco (Laboratoire Charles Coulomb CNRS, France) was motivated by self-propelled motion of catalytic Janus colloids at the air-water interface. Active motion is not expected to occur if either the catalytic surface is completely out of the aqueous phase or the Janus boundary is parallel to the liquid interface. As a prerequisite for active propulsion, the paper therefore considers the wetting and orientation of Janus particles at the air/water interface (DOI:

$10.1039 / \mathrm{c} 6 \mathrm{fd}$ 00025h). From minimum energy calculations, the authors demonstrate it is not possible to use amphiphilic Janus colloids to obtain the required orientation for active motion, and one should instead use double hydrophilic Janus colloids. However, a key take-home message of the paper is that contact line pinning strongly modifies the behaviour of Janus colloids away from the ideal scenario described above. In particular, contact line pinning can lead to the Janus colloids adopting orientations that are energetically unfavourable at the air/water interface and also strongly decrease (by an order of magnitude) the rotational and translational diffusion of the particles at the air/water interface.

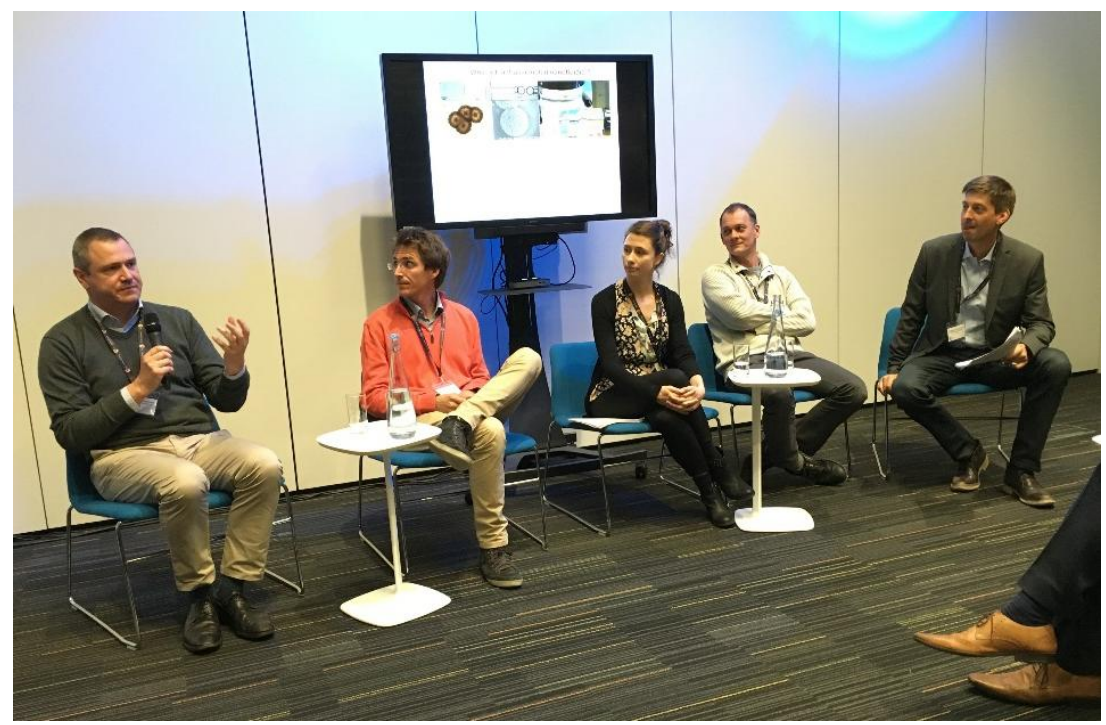

Fig. 2: Some of the participants of the third session. From left to right, Prof Jan Vermant, Dr Matthias Pauly, Dr Estefania Gonzalez Solveyra, Dr Antoine Thill, Prof Joerg Lahann. Photo courtesy of Luis Liz-Marzán 
After the afternoon coffee break, the session continued with an interesting paper by Prof Jan Vermant (ETH Zurich, Switzerland) who revisited the synthesis of the quintessential model system for hard spheres, i.e., sterically stabilised PMMA particles (DOI: 10.1039/c6fd00052e). The synthesis method for this system is well known but also notoriously difficult to control. Through extensive use of ${ }^{1} \mathrm{H}$ NMR to monitor the molecular weight of the reaction products, the authors were able to trace the problem back to the difficulty in synthesising the graft stabiliser (PMMA-g-PHSA). By changing the catalyst and performing the polycondensation of PHSA in the melt phase, the authors were able to gain much better control of the synthesis of PHSA and hence the PMMA hard spheres. Finally, by using a range of mechanical deformation methods, the authors show that it is possible to use the PMMA spheres to create micron sized colloids with anisotropic shapes such as ellipsoids and surfboards. The latter shape is particularly interesting with regards to their in-plane shear alignment properties at a liquid interface.

In a beautiful paper, Dr Estefania Gonzalez Solveyra (Northwestern University, USA) considered how one could exploit the differential curvature between the cylindrical body and spherical end caps of gold nanorods to create end-functionalised nanorods (DOI: 10.1039/c6fd00020g). Specifically, the authors construct a molecular theory to investigate the equilibrium adsorption of a mixture of linear and branched polymers on the body and end-caps of the nanorod, taking into account both polymerpolymer and polymer-surface interactions. Through a systematic study of polymer adsorption as a function of the composition, size, architecture and solvent quality of the polymer mixture, the authors found suitable conditions where the end caps are significantly enriched with the branched species (due to greater available volume around at the end caps). The study therefore suggests a novel method for creating chemically inhomogeneous gold nanorods driven by surface curvature.

Dr Matthias Pauly (Institut Charles Sadron CNRS, France) described a simple but effective method for aligning anisotropic metallic particles on a solid substrate using spray-assisted assembly (DOI: $10.1039 / \mathrm{c} 6 \mathrm{fd} 00017 \mathrm{~g})$. Specifically, the alignment of the anisotropic particles is achieved by spraying droplets of the nanoparticle suspension at low grazing angles, and the alignment was found to increase with increasing aspect ratio of the nanoparticles. In particular, the authors were able to use this method to create oriented silver nanowire monolayers where the sheet resistance varies by over an order of magnitude depending on the transport direction.

The final paper in the session by Dr Antoine Thill (CEA Saclay, Universite Paris-Saclay, France) considered the fascinating system of Imogolites, naturally occurring nanotubes with a diameter of a few nanometers but a length of several microns (DOI: 10.1039/c6fd00034g). Because Imogolites have different chemistries on the internal and external surfaces, the authors were able to modify these systems to prepare Imogolites with a hydrophobic external surface and a hydrophilic cavity (OPA-imo) and those with a hydrophilic external surface and a hydrophobic cavity (imo- $\mathrm{CH}_{3}$ ). By filling the Imogolites with the appropriate internal solvent, the authors found that OPA-imo and imo- $\mathrm{CH}_{3}$ could be used to stabilise water-in-oil and oil-in-water emulsions respectively. Remarkably, OPA-imo adopts a perpendicular orientation at the oil/water interface of the water droplets, and it is postulated that this orientation is stabilised by the lateral interactions between neighbouring nanotubes. On the other hand, there is evidence of small molecule transport inside imo- $\mathrm{CH}_{3}$, suggesting possible applications in self-assembled nanofluidics.

After the session, the conference attendees gathered at the Glasgow Town Hall to enjoy drinks in a beautiful historical venue. Bailie Eva Bolander, from the Glasgow city council, gave a speech highlighting the willingness of the city of Glasgow to be the host of such events and to be a friendly city for science and the scientists altogether. The conference dinner then took place and enabled further discussion between the delegates. In the middle of the dinner, the traditional Loving cup ceremony was introduced by Pr Eleanor Campbell, president of the Faraday Divison at the RSC. This 
traditional feature of the Faraday conferences consists in drinking Port wine in a $18^{\text {th }}$ century silver cup while respecting a precise protocol to pass the cup between the guests. This protocol includes the toast "In piam memoriam of GS Marlow and Angela \& Tony Fish" in memory of past members of the Faraday society.

\section{Session 4 Applications}

The fourth session took place on the morning of 6 July, with Prof. Molly Stevens (Imperial College London) as session chair. The session's main topic revolved around functionalized nanoparticles that are applicable in biological or medical fields. Dr. Yarovsky spoke about the theoretical study of surface adsorption behavior of proteins, while other speakers focused on the relationship between synthetic approaches and anisotropy of nanoparticles that are created.

Prof Irene Yarovsky (RMIT University, Melbourne, Australia) was the first presenter, discussing the theoretical study of surface adsorption of the protein EAS hydrophobin (DOI: 10.1039/c6fd00050a).

Six systems of functionalized surface were simulated by bonding different functional groups to a simulated amorphous silica surface. Hydrolysed glycidoxypropylsilane functional chains were used to represent the hydrophilic systems, and fluorinated chains represented hydrophobic surfaces. All-atom MD simulations were used to examine the adsorption for EAS hydrophobin on these six systems. It was concluded that the nanoscale chemical patterning and surface roughness of the functional chain length are key influencing factors for protein adsorption. It was confirmed that EAS is a versatile protein which can adsorb reasonably well to all surfaces. Interestingly, the highly flexible amphipathic Cys3-Cys4 loop in the protein is important for stabilizing protein adsorption, and for interacting with other EAS proteins to form monolayers.

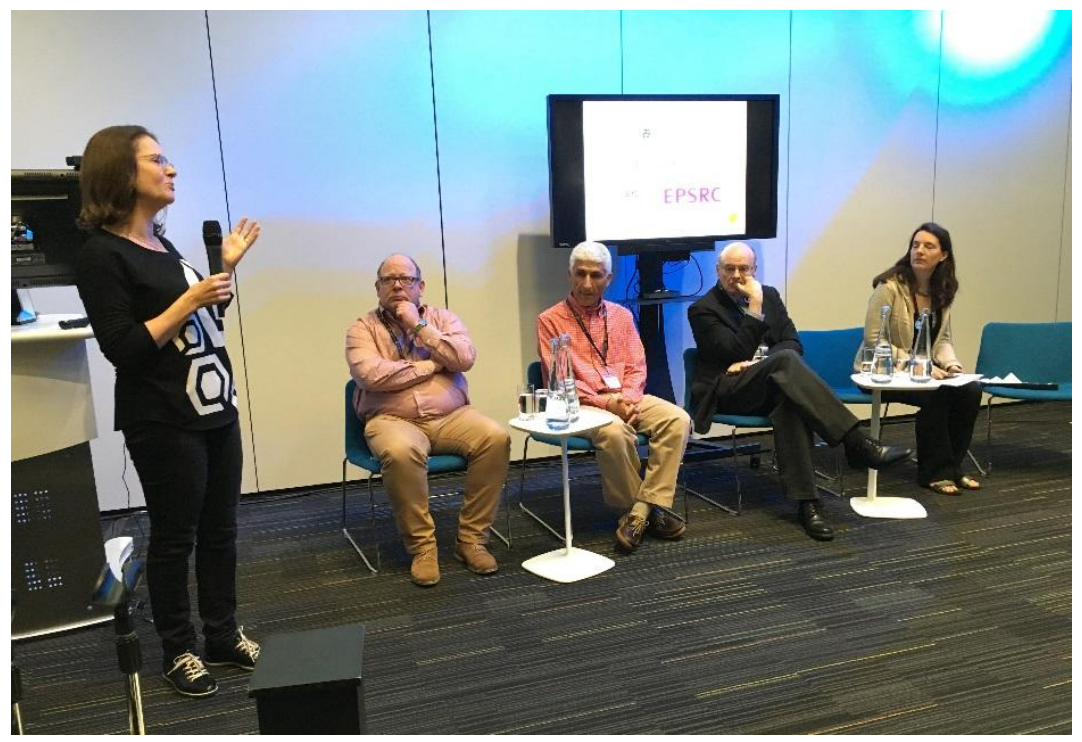

Fig.3: Some of the participants of the fourth session. From left to right, Prof Irene Yarovsky, Prof Mathias Brust, Prof Hedi Mattoussi, Prof Richard Jones, Prof Molly Stevens. Photo courtesy of Luis Liz-Marzán

Prof Richard Jones (The University of Texas at Austin, USA) talked about the self-assembly of lanthanide-based nanoclusters using Schiff bases (DOI: 10.1039/c6fd00018e). Seven d-f clusters were prepared using different Schiff base ligands with flexible carbon chains. Discussions focused on the architecture of nanoclusters, with Cd-Ln clusters in nano-drum-like structures, and the Ni-Ln clusters 
having square structures. The nanoclusters exhibit NIR luminescence properties, which is useful in bio-imaging. The mechanism for the self-assembly was discussed during the discussion session.

Prof Hedi Mattoussi (Florida State University, Tallahassee, USA) reported on the synthesis of a multicoordinating polymer ligand (lipoic acid- and PEG-modified PIMA polymer) which could be used for functionalizing gold nanoparticles and nanorods (DOI: 10.1039/c6fd00056h). The polymer was prepared using nucleophilic addition reaction, with a poly(isobutylene-alt-maleic anhydride) precursor. Oleylamine-capped AuNPs and CTAB-capped AuNRs underwent ligand exchange with the synthesized polymers, which yielded nanocrystals that exhibit excellent long-term colloidal stability.

Prof Mathias Brust (University of Liverpool, UK) presented his research on tunable hydrophobicity of gold nanoparticles (DOI: 10.1039/c6fd00037a). A 18-crown-6- $\mathrm{CH}_{2}$-thiolate ligand shell with potassium ions were used as a capping agent for the gold nanoparticles. This capping agent is the key of controlling hydrophobicity. The phase transfer of these particles from dispersion in water to chloroform was demonstrated. Using cryo-TEM, it was observed that the nanoparticles could be used to insert into vesicle membranes in an aqueous dispersion. It is concluded that the nanoparticles can be used as membrane transporters.

Dr. David Portehault (CNRS, Sorbonne Universités-UPMC Univ., France) presented his research on the synthesis of metal borides using the molten salt method (DOI: 10.1039/c6fd00053c). This is the first time metal borides have been synthesized using inorganic molten salts. It was highlighted that the method is relatively simple, requires low temperature, and the stoichiometry of the metal boride nanocrystals can be tuned simply by changing the amount of reagents. Two metal-boron systems were studied: the boron-poor nickel borides and boron-rich yttrium borides. It was observed that the two systems exhibited anisotropic crystallization, but the Ni-B system crystallized as spheres, while the YB system crystallized as rods. Molten salt synthesis has been of interest in the research of functional nanomaterials, and this study is a good example of fabricating nanocrystals using the method. The mechanisms of the reactions and the crystal formation was a focal point in the discussion. It was speculated that the crystal formation depends largely upon the heating/cooling process. For Y-B, the morphology of the crystal followed the Wulff construction, which states that a droplet or crystal will rearrange itself in such a way that the surface energy is minimized, hence the nanorod structure. The Ni-B takes up a spherical morphology due to the constrains within the structure, and it was speculated that tuning the rate of the cooling process may cause the morphology to change.

Prof Lucia Pasquato (University of Trieste, Italy) gave a talk on grafting mixed monolayers of fluorinated and hydrogenated alkanethiolates on the surface of gold nanoparticles (DOI: 10.1039/c6fd00016a). Mixed monolayer gold nanoparticles (MMNPs) were prepared using fluorinated and hydrogenated ligands. The behaviour of the MMNPs corresponding to the different domains of the particles were a point of discussion.

Prof Calum Drummond (RMIT University, Melbourne, Australia) presented the study on synthesis of amphiphilic brush polymers, using Reversible Addition-Fragmentation chain Transfer (RAFT) polymerization, and stabilizing and cytotoxicity reduction of lipid lyotropic liquid crystalline nanoparticles (LCNPs) (DOI: 10.1039/c6fd00039h). LCNPs can be applied in drug delivery, which requires the NPs to be stabilized and flexible, to achieve optimal encapsulations and targeting functions. The RAFT technique was used to synthesize brush amphiphilic polymers consisting of a C12 short chain and a poly(ethylene glycol)methyl ether acrylate long chain, which were then used as stabilizers to formulate phytantriol or monoolein-based LCNPs. The internal lyotropic liquid crystalline phase and the cellular tolerance of the formulated nanoparticles were investigated in vitro. The techniques employed were synchrotron small-angle X-ray scattering (SAXS) and cryo-TEM. It was found that the RAFT synthesized P(PEGA) amphiphilic brush polymers are comparable to Pluronic block copolymers as stabilizers. The brush polymers provides effective colloidal stabilization, and demonstrated a marked improvement in cellular tolerance towards phytantriol-based cubosomes, 
compared with Pluronic polymers. This work draws interest for using non-commercial polymers as stabilizers for LCNP drug delivery systems.

\section{Perspective lecture:}

Prof Axel H.E. Müller, from Johannes Gutenberg Universität in Mainz, presented an invited perspective lecture describing recent work of his group on the synthesis and self-assembly of ABC block copolymers into original nanostructure. Prof Müller provocatively entitled his talked "Is there a life beyond inorganic or hybrid materials ?" to stress that most of the work presented during the conference dealt with inorganic nanoparticles. Of course, considering the beauty of the structures displayed during his talk, everybody was convinced that, indeed, organic materials made of copolymers have a bright future! The research presented is based on the synthesis of triblock copolymer with designed architectures which serve as soft colloidal building blocks for higher level self-assembled structures. The physical properties and the shape of these particles can be finely tuned to yield Janus particles with varying size and hydrophilic/hydrophobic balance. To do so, Prof Müller and his group selectively cross-link certain parts of multicompartment micelles formed by the selfassembly of the tri-block copolymers in selective solvents. For example, an ABC copolymer selfassembles into precisely shaped micelles in a non-solvent for $\mathrm{A}$ and $\mathrm{B}$, forcing the $\mathrm{C}$ component to point out towards the solution and retaining the other blocks inside the micelle. The B part can then be cross linked using UV light, hence 'freezing in' this part of the structure. When the micelles are dispersed in a good solvent for all the blocks, they disassemble yielding Janus particles. Once these particles are made, they can, in turn, self-assemble into larger structures, forming micelles of particles. The shape of these super-particles is encoded in the valence and patchy character of the initial colloidal building blocks. Spherical clusters or long chains resembling colloidal polymers can form depending on whether the particle is initially monovalent or divalent. Finally, mixing two different types of soft patchy particles with distinct sizes and patchiness can yield co-assembled binary hierarchical superstructures. Particularly impressive is the degree of control of the hierarchical structures at all length scales ranging from the atomic control of the triblock copolymer to the nanometer scale with the micelles towards the micrometer scale with the assembly of compartmentalized, substructured materials. Such a finely tuneable complexity over three orders of magnitudes in size is not achieved yet for inorganic materials but should inspire current research as a long term goal to reach.

\section{Concluding remarks lecture}

To conclude the conference, the attendees were fortunate to listen to Prof Younan Xia from Georgia Tech University (USA) who talked about the emergence of anisotropy in metallic nanoparticles. Before detailing recent results obtained in his group, Prof Xia exposed general concepts on anisotropy related to the nature of the building blocks. The propensity of the different atomic elements to construct anisotropic structures is ultimately encoded into the geometry of its bonding electronic orbitals. For example, the fact that carbon is so ubiquitous could be related to the fact that this is the element with the minimal number of electrons having the potential to bond in three different directions through it $\mathrm{p}$ orbitals. He stressed that, contrary to covalent bonding, metallic bonding in not directional which makes it difficult to produce anisotropic particles made of metals. Hence, a combination of internal and external factors must be used to tune anisotropy in such systems. Twin defects, stacking faults or nucleation of a certain type of nuclei can be internal causes to the emergence of anisotropy. On the other hand, tuning the kinetics of the precursor chemistry or the use of a dedicated capping agent are two knobs that one can act on in order to induce the emergence of anisotropy. To illustrate the latter concept, Prof Xia showed that the shape of silver nanocrystals could be changed from cubes with exposed $\{100\}$ facets to octahedrons enclosed by $\{111\}$ facets by changing the capping agent from sodium citrate to poly(vinyl pyrrolidone). It was also stressed that, 
since nanoparticle synthesis is most of the time kinetically controlled, an initial symmetry breaking can persist as the synthesis proceeds. A simple way to achieve this is to limit the supply of atoms during the growth phase so that only certain favoured facets grow. Depending on the injection rate of silver precursors on palladium nanocubes, different geometries can be obtained. Fast injection favors isotropic growth where all the facets of the initial seeds are equally covered with $\mathrm{Ag}$, leading to a core shell geometry. As the injection rate diminishes, growth occurs on less and less facets and ultimately, $\mathrm{Ag}$ only grows on one face of the initial cube to form hybrid dimers. Precursor kinetics can also rationalize shape control in the synthesis of palladium nanocrystals. Starting from cuboctahedral seeds, two different precursors with different reaction rates yield either octahedrons or tetrahedrons. When a fast precursor is used, only $\{100\}$ facets grow and the cuboctahedron transforms into octahedrons and then tetrahedrons. In contrast, the use of a slower precursor enables the concomitant growth of all the eight $\{111\}$ facets resulting in the formation of octahedrons which grow in size as the slow reaction proceeds.

Along the same line of research, Prof Xia then presented impressive results on the quantitative understanding of symmetry reduction in the seed mediated growth of Pd nanocrystals. By using a syringe pump to slowly introduce the precursors in a solution containing seeds, it is possible to achieve a constant concentration of precursor ion in solution. Two growth modes were identified: a symmetric mode where all the facets of the initial seed grow and an asymmetric mode where only certain, more reactive facets grow. Interestingly, to sustain asymmetric growth, the rate at which metallic atoms are brought in solution has to outpace surface diffusion. On the contrary, atoms deposit on specific facets but surface diffusion blurs this initial symmetry breaking. On the other, the initial symmetry breaking occurs only if the the first few drops do not bring too much material in solution as compared to the amount of seeds. This shows that a delicate balance between initial seed ratio, temperature and injection speed has to be found to yield desired product.

Overall, Prof Xia's talk brilliantly demonstrated that the fundamental mechanisms of shape control in metallic nanocrystals have now begun to be understood in a quantitative fashion. This will provide guidance in the experimental conditions that are necessary to achieve fine control over the diversity of potential shapes. In the perspective of synthesizing precisely shaped particles for targeted applications in plasmonics or catalysts, this mechanistic work will be of huge relevance. 


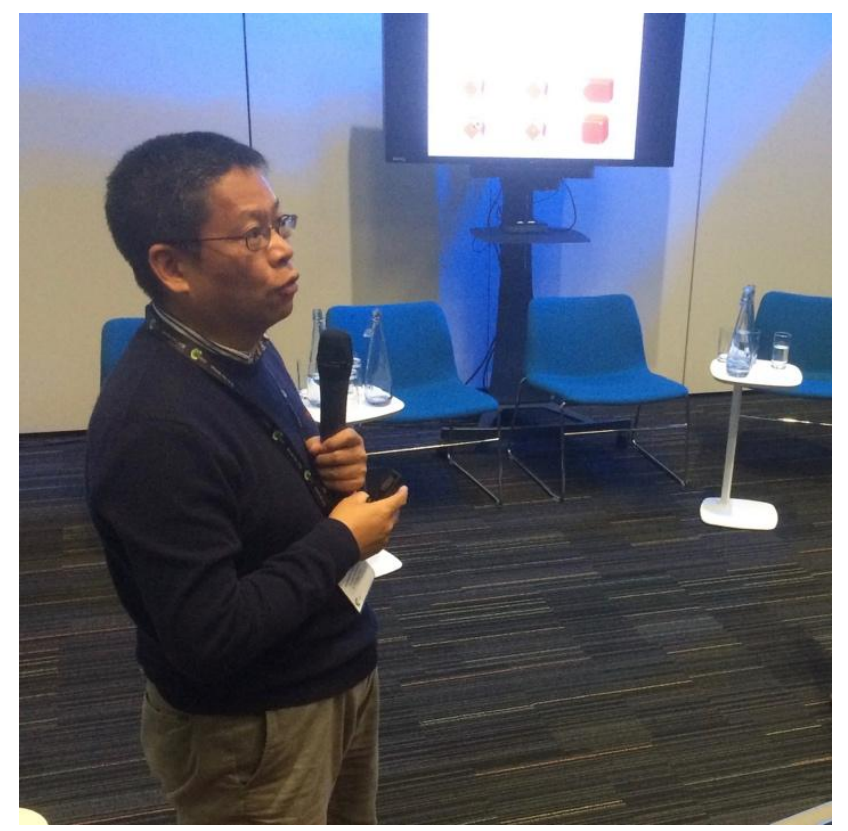

Figure 4: Pr Younan Xia presenting his closing lecture. Photo courtesy of Benjamin Abécassis.

After closing remarks from the Chair of the conference, the delegates cheerfully thanked the organizers for their outstanding work and shared a last lunch before going back home. 